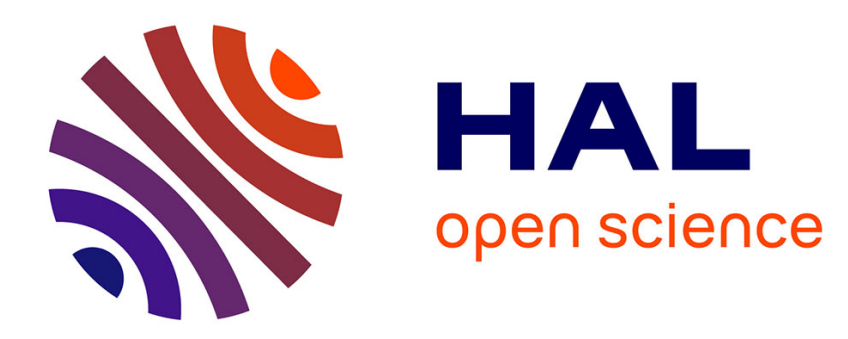

\title{
A static interpretation of the order-parameters in spin-glasses
}

\author{
Henri Orland
}

\section{To cite this version:}

Henri Orland. A static interpretation of the order-parameters in spin-glasses. Journal de Physique Lettres, 1983, 44 (16), pp.673-677. 10.1051/jphyslet:019830044016067300 . jpa-00232248

\section{HAL Id: jpa-00232248 https://hal.science/jpa-00232248}

Submitted on 1 Jan 1983

HAL is a multi-disciplinary open access archive for the deposit and dissemination of scientific research documents, whether they are published or not. The documents may come from teaching and research institutions in France or abroad, or from public or private research centers.
L'archive ouverte pluridisciplinaire HAL, est destinée au dépôt et à la diffusion de documents scientifiques de niveau recherche, publiés ou non, émanant des établissements d'enseignement et de recherche français ou étrangers, des laboratoires publics ou privés. 
Classification

Physics Abstracts

$64.60 \mathrm{C}-64.60 \mathrm{M}-75.50 \mathrm{~K}$

\title{
A static interpretation of the order-parameters in spin-glasses
}

\author{
H. Orland \\ Institute for Theoretical Physics, University of California, Santa Barbara, CA 93106, U.S.A. and \\ Service de Physique Théorique (*), CEN-Saclay, 91191 Gif-sur-Yvette Cedex, France
}

(Reçu le 22 novembre 1982, révisé le 21 avril 1983, accepté le 20 juin 1983)

\begin{abstract}
Résumé. - La phase verre de spin est décrite comme un mélange statistique d'un nombre infini de phases. Les paramètres d'ordre $q(x)$ et $\Delta(x)$, avec $x \in[0,1]$, mesurent respectivement les corrélations entre ces phases et l'anomalie de réponse linéaire. La solution de Sompolinsky est ainsi obtenue, et rediscutée.
\end{abstract}

Abstract. - The spin-glass phase is described as a statistical mixture of an infinite number of phases.
The order-parameters $q(x)$ and $\Delta(x)$, with $x \in[0,1]$ measure the correlation between the phases, and
the linear response anomaly respectively. Sompolinsky's solution is recovered and reinterpreted.

The nature of the order-parameter of the spin-glass (SG) phase has been a long-standing puzzle [1, 2]. Two different approaches have recently been proposed. The first, due to Parisi [3], involves an order-parameter function $q(x)$ with $x \in[0,1]$. It involves replicas, and its physical meaning as well as its mathematical derivation remains mysterious. The second approach, due to Sompolinsky [4], is a dynamical one. It assumes that relaxation to equilibrium proceeds through an infinite hierarchy of relaxation times $t_{x}$, and the order parameters appear as the correlation function $\overline{\left\langle S(0) S\left(t_{x}\right)\right\rangle}$ at time scale $t_{x}$ (where $\langle\cdots\rangle$ and $\cdots$ mean respectively thermal and disorder average). It implies a complete decay of the SG ordering in the static limit. It turns out that these two approaches are essentially equivalent [5], and are acknowledged as the correct mean-field theory for SG [6].

The non-ergodicity of the infinite-range Ising spin-glass has been recognized recently [7]. As a consequence, one expects time averages not to be equal to ensemble averages, and to depend on the time scales considered [8]. Although we believe that ultimately, the physics of SG is to be described by dynamics rather than thermodynamics, it may be interesting to understand the infinite-range model in terms of purely static, or thermodynamical concepts.

(*) Permanent address. 
The purpose of this paper is to provide such an interpretation; the following points are shown :

i) First, it is possible to give a physical meaning to replicas, and even more, to remove the ambiguity in the analytic continuation at $n=0$, provided one follows a well-defined procedure which is discussed in the text. The replicas are shown to represent the statistical composition of the system in thermodynamic equilibrium. In particular, symmetry-breaking is shown to be directly related to the coexistence of different phases in the system.

ii) In the infinite-range Ising SG, where an infinite number of phases [12] (interpreted as solutions of the Thouless-Anderson-Palmer [9] equations) is available to the system, we show that all these phases have the same Edwards-Anderson (EA) order parameter [1], $q_{\mathrm{EA}}=\frac{1}{N} \sum_{i} \overline{\left\langle S_{i}^{2}\right\rangle}$. The Parisi ansatz [3], which is discussed in this paper, is shown to describe the system as a statistical mixture of all these phases, the order-parameter $q(x)$ measuring the correlation or overlap between these phases. This does not imply that the Parisi construction is the only possible scheme, but it shows that it is a physically sensible one.

Replicas are easily interpreted using the microcanonical ensemble. Consider the microcanonical density :

$$
\rho(E)=\sum_{\left\{S_{i}\right\}} \delta\left(E+\sum_{i j} J_{i j} S_{i} S_{j}\right)
$$

where $\sum_{\left\{\boldsymbol{S}_{i}\right\}}$ denotes a sum over spin configurations.

The canonical ensemble, as originally presented by Gibbs, is obtained by replicating the system a large number of times $M$, the total energy being fixed. The density of states for this composite system reads :

$$
\rho_{M}\left(E_{0}\right)=\int \prod_{p=1}^{M} \mathrm{~d} E_{p} \rho\left(E_{p}\right) \cdot \delta\left(E_{0}-\frac{1}{M} \sum_{p=1}^{M} E_{p}\right) .
$$

which can be rewritten :

$$
\rho_{M}\left(E_{0}\right)=\int \frac{\mathrm{d} \lambda}{2 \Pi / M} \exp \left[M\left(i \lambda E_{0}+\log \left(\int \mathrm{d} E \mathrm{e}^{-i \lambda E} \rho(E)\right)\right)\right] .
$$

When $M \rightarrow+\infty$, it is calculated by the saddle-point method (SPM) :

$$
E_{0}=\frac{\int \mathrm{d} E \rho(E) \mathrm{e}^{-i \lambda E} E}{\int \mathrm{d} E \rho(E) \mathrm{e}^{-i \lambda E}}
$$

which is to be solved for $i \lambda$, which is the physical temperature $\beta$. The density of states is then :

$$
\rho_{M}\left(E_{0}\right) \underset{M \rightarrow+\infty}{\sim} \mathrm{e}^{M S\left(\beta\left(E_{0}\right)\right)}
$$

where $S\left(\beta\left(E_{0}\right)\right)$ is the canonical entropy of any subsystem.

The $M$ subsystems indexed by $p$ represent the different fluctuation states of a canonical system at temperature $\beta$. All the subsystems have average energy $E_{0}$, and they fluctuate independently around $E_{0}$. In a situation of broken symmetry different phases have the same energy, and there- 
fore each subsystem condenses independently in any of these phases. The system at temperature $\beta$ appears as a statistical mixture of these phases, but the composition of the system in the different phases is not fixed. It is a situation of a first order transition, with different phases coexisting in the system.

We now turn specifically to the Sherrington-Kirkpatrick (SK) [10] model. Equation 5 has to be averaged over the disorder :

$$
\overline{\rho_{M}(E)} \underset{M \rightarrow+\infty}{\sim} \overline{\mathrm{e}^{M S(\beta(E))}} .
$$

In order to get physical quenched averages, one must take a derivative of (6) with respect to $M$ and then set $M=0$. This seems like an old disease, however, in the present case, we can show that the analytic continuation at $M=0$ is unique. Indeed, for any configuration of the bonds,

$$
S(\beta)<N \log 2
$$

and therefore, when $M \rightarrow+\infty$

$$
\rho_{M}(E)<2^{N M}
$$

Therefore, for any complex number $n$, with $\operatorname{Re} n>0$, we have (when $M \rightarrow+\infty$ )

$$
\overline{\left|\rho_{M}^{n}(E)\right|}<2^{N M R e n} .
$$

According to Carlson's theorem [11], analytic continuation of the function $\overline{\rho_{M}^{n}(E)}$ defined now for integer $n$ is unique. Since for any integer $n$, we have :

$$
\overline{\rho_{M}^{n}(E)} \underset{M \rightarrow+\infty}{\sim} \overline{\rho_{n M}(E)}
$$

we see that in order to compute quenched averages, we must proceed in the following way :

i) Compute $\overline{\rho_{n M}(E)}$ for integer $n M$.

ii) Take the limit $M \rightarrow+\infty$, to build up a statistical ensemble representative of the system at temperature $T$

iii) Then take the limit $n \rightarrow 0$.

For the SK model, the average over a Gaussian distribution of bonds yields

$$
\begin{aligned}
\overline{\rho_{n M}(E)}=\int \frac{\mathrm{d} \beta}{2 \Pi / n M} \cdot \prod_{1 \leqslant p<q \leqslant n M} \frac{\mathrm{d} p_{p q}}{\sqrt{2 \Pi / N}} \exp \left[n M\left(\beta E+N \frac{\beta^{2} J^{2}}{4}\right)\right] \times \\
\quad \times \exp \left(-\frac{N}{2} \sum_{1 \leqslant p<q \leqslant n M} q_{p q}^{2}\right)+N \log \left(\operatorname{Tr}_{\left\{S_{p}\right\}} \exp \left[\beta J \sum_{1 \leqslant p<q \leqslant n M} q_{p q} S_{p} S_{q}\right]\right) .
\end{aligned}
$$

In the thermodynamic limit, (8) can be calculated by using the SPM :

$$
q_{p q}=\beta J\left\langle S_{p} S_{q}\right\rangle
$$

and

$$
E=-\frac{N \beta J^{2}}{2}-\frac{N}{\beta M n} \sum_{p<q} q_{p q}^{2}
$$

Equation $9 a$ shows clearly the effect of the disorder, it correlates the different subsystems. 
If different phases are available the subsystems will condense in these phases, in such a way as to satisfy equation $9 a$.

It has been shown that the total number of solutions of the Thouless-Anderson-Palmer (TAP) equations behave like $\mathrm{e}^{\bar{\alpha} N}$ [12]. Therefore, the total number of phases available at temperature $T$ is exponentially large. It is easily seen that all these phases have the same EA order parameter. Indeed, the number of solutions of the TAP equations with EA order parameter $q_{0}$ is given by :

$$
n\left(q_{0}\right)=\int \prod_{i=1}^{N} \mathrm{~d} m_{i} \prod_{i} \delta\left(\frac{\partial F\left(m_{i}\right)}{\partial m_{i}}\right) \cdot \operatorname{Det}\left(\frac{\partial^{2} F\left(m_{i}\right)}{\partial m_{i} \partial m_{j}}\right) \delta\left(q_{0}-\frac{1}{N} \sum_{i} m_{i}^{2}\right)
$$

where $F\left(m_{i}\right)$ is the TAP free-energy.

A calculation identical to the one of reference 12 shows that if $q_{0}=q_{\mathrm{EA}}(\beta), \overline{n\left(q_{0}\right)}=\mathrm{e}^{\alpha N}$, whereas if $q_{0} \neq q_{\mathrm{EA}}(\beta), \overline{n\left(q_{0}\right)}=0$. (This is trivial at $T=0$, where all solutions have $q_{\mathrm{EA}}=1$ ). It can similarly be shown that the correlation $q_{12}$ between two phases can very continuously (in the thermodynamic limit) between $q_{\mathrm{EA}}$ (when the two phases are identical) and 0 . A direct approach starting from the TAP equations will be reported elsewhere [13].

The effect of frustration and disorder can now be clearly separated :

i) Frustration enables the existence of an infinite number of phases, which all have the same EA order parameter.

ii) Disorder correlates these phases, and forces them to condense simultaneously according to a certain pattern of correlation $q_{p q}$.

Since $q_{p q}$ is the correlation of the magnetization of states $p$ and $q$, it measures the number of spins which are different in states $p$ and $q$; it is a distance, in configuration space between these states.

Any theory which does not take full account of this infinite number of phases is expected to describe metastable states. Such is the case of the SK solution [10], where taking all $q_{p q}$ equal amounts to describe the system as a single phase.

In order to specify the system, we must label the phases. Since all the phases have same EA order parameter, we classify them in a hierarchical way, by decreasing order of correlation. It amounts to taking a correlation matrix of the Parisi type, namely

$$
q_{p q}=\left(\begin{array}{c|c|c}
q_{1} & q_{0} & q_{0} \\
\hline q_{0} & q_{1} & q_{0} \\
\hline q_{0} & q_{0} & q_{1}
\end{array}\right)
$$

where the size of the subblocks is $p_{1}$, then iterate a Parisi type of procedure on the diagonal blocks, i.e. break each diagonal block into a structure similar to (11), with diagonal blocks $q_{2}$ of size $p_{2}$ and unchanged off-diagonal blocks, etc...

One ends up with a hierarchy of blocks of size $n M=p_{0}>p_{1}>\cdots>p_{k}$, and correlation $0=q_{0} \leqslant q_{1} \leqslant q_{2} \leqslant \cdots \leqslant q_{k}=q_{\mathrm{EA}}$.

The diagonal blocks of size $p_{k}$, with $q_{k}=q_{\mathrm{EA}}$, represent the phases present in the spin glass. All other blocks represent the correlations between these phases. It follows from the TAP equations that one has necessarily $p_{0} \gg p_{1} \gg \cdots \gg p_{k}$. Indeed, one can show that if $q_{l+1}>q_{l}$, the numbers of pairs of phases with correlation $q_{l}$ is exponentially larger than the number of pairs with correlation $q_{l+1}$ [13].

Following the procedure of reference 5 , we define $x=l / k, l=1, \ldots, k$, and $q_{l}-q_{l-1}=$ $q^{\prime}(x) \mathrm{d} x-\Delta^{\prime}(x) / p_{l} \mathrm{~d} x$, where $\Delta^{\prime}(x)$ is the linear response anomaly. When $M \rightarrow+\infty$, all the $p_{l}$ go to infinity in the order prescribed above, and it is easily seen that after performing the last step, i.e. $n \rightarrow 0$, one recovers Sompolinsky's solution. 
The physical interpretation of this solution is the following : the system is a statistical mixture of an infinite number of phases. All these phases are thermodynamically equivalent, and have the same EA order parameter $q_{\text {EA }}$. They can be grouped hierarchically, by decreasing order of correlation, and the correlation function between these phases is characterized by the orderparameter $q(x)$ [14]. Since the system is described as a statistical mixture, a dynamical picture is easy to draw if we could take " instantaneous pictures " of the system at equilibrium at different times, these phases would appear with frequencies such as to build up correlations characterized by the order parameter $q(x)$.

Also, the linear response anomaly is the result of the infinite number of phases present in the system, indeed, applying a magnetic field to a given phase induces a magnetization in all other phases present in the system (since they are correlated). Finally, within this interpretation, the Thouless-Almeida instability line [2] can be interpreted as the line of transition from a multiphase to a monophase system.

\section{Acknowledgments.}

The author wishes to thank C. De Dominicis and H. Hartman for helpful discussions. This research was supported by NSF grant \#PHY77-27084.

\section{References}

[1] Edwards, S. F., ANderson, P. W., J. Phys. F 5 (1975) 965.

[2] De Almeida, J. R. L., Thouless, D. J., J. Phys. A 11 (1978) 983.

[3] PARISI, G., Phys. Rep. 67 (1980) 25 and references therein.

[4] Sompolinsky, H., Phys. Rev. Lett. 47 (1981) 935.

[5] De Dominicis, C., Gabay, M., Duplantier, B., J. Phys. A 15 (1982) L-47.

[6] Thouless, D. J., De Almeida, J. R. L., Kosterlitz, J. M., J. Phys. C 13 (1980) 3271.

[7] Mackenzie, N. D., Young, A. P., Phys. Rev. Lett. 49 (1982) 301.

Young, A. P., KirkPatrick, S., Phys. Rev. B 25 (1982) 440.

Toulouse, G., in Anderson Localization, Proceedings of the 1981 Taniguchi Symposium, Springer Series in Solid-State Sciences, Vol. 39 (Springer, Berlin, to be published).

[8] Young, A. P., J. Phys. C 14 (1981) L-1085.

[9] Thouless, D. J., Anderson, P. W., Palmer, R., Philos. Mag. 35 (1977) 593.

[10] Sherrington, D., KirkPatrick, S., Phys. Rev. Lett. 35 (1975) 1792.

[11] Van Hemmen, J. L., Palmer, R. G., J. Phys. A 12 (1979) 563.

[12] Bray, A. J., Moore, M. A., J. Phys. C 13 (1980) L-469.

De Dominicis, C., Gabay, M., Garel, T., Orland, H., J. Physique 41 (1980) 923.

Tanaka, F., Edwards, S. F., J. Phys. F 10 (1980) 2471.

[13] De Dominicis, C., OrLand, H., to be published.

[14] This interpretation of $q(x)$ as a correlation between phases has also been discussed by HougHTON, A., Fain, S. and Young, A. P., J. Phys. C 16 (1983) L-375 and by Parisi, G. (Preprint). 